\title{
Raman Scattering and the Hidden He II Emission Region in the Bipolar Planetary Nebula M2-9
}

\author{
Hee-Won Lee \\ Dept. of Geoinformation Sciences, Sejong University, Seoul, Korea
}

\begin{abstract}
In the spectrum of the young bipolar planetary nebula M29, we detected the He II Raman scattered feature at $6545 \AA$. However, in the same spectrum the He II emission lines at $6527 \AA$ and $6560 \AA$ are absent, which implies that the He II emission region is hidden from our line of sight and that the $\mathrm{H} \mathrm{I}$ scattering region is pretty much extended not to be obscured entirely. We perform photoionization computations to estimate the physical size of the He II emission line region $\lesssim 10^{15} \mathrm{~cm}$ in the presence of hot luminous star with $T \gtrsim 10^{5} \mathrm{~K}$ and $L \gtrsim 10^{3} L_{\odot}$.
\end{abstract}

\section{Observation}

The young and prototypical bipolar planetary nebula M2-9 is known to exhibit very broad wings around $\mathrm{H} \alpha$ (e.g. Balick 1989). The wing formation mechanism is not clear currently and Lee (2000) proposed that the Raman scattering of Ly $\beta$ can produce broad wings around $\mathrm{H} \alpha$ (Schmid 1989). The bipolar morphology is believed to be strongly associated with the dense circumstellar region, which is conspicuous in the infrared and radio spectral regions. It is difficult to study the heavily obscured central star. Recent studies show that the bipolar morphology may be closely connected with the fact that the central star forms a binary system (Doyle et al. 2000).

Using the Bench-Mounted Echelle spectrometer installed on the CTIO 1.5 $\mathrm{m}$ telescope, we obtained high resolution spectra of M2-9 and several symbiotic stars in order to study the operation of Raman scattering in these objects. Fig. 1 shows the spectra of M2-9 and the symbiotic star RR Telescopii around $\mathrm{H} \alpha$. From this figure, we note that in RR Tel there are two He II emission lines at 6560 $\AA$ arising from the transition $n=6 \rightarrow n=4$ and $6527 \AA$ from $n=14 \rightarrow n=5$. However, these emission lines are absent in M2-9. In both objects, we detect a feature at $6545 \AA$ which can be clearly isolated after subtraction of [N II] 6548 using [N II] 6584 multiplied by 1/3 (Lee, Kang \& Byun 2000).

The $6545 \AA$ feature is proposed to be the Raman scattered line of He II $1025(n=6 \rightarrow n=2)$ by atomic hydrogen. The right panel of Fig. 1 illustrates the Raman scattering process and a simple calculation shows that the cross section is $\sigma \sim 10^{-20} \mathrm{~cm}^{2}$. The detection of this feature implies that there exist a He II emission region in both objects surrounded by a thick neutral hydrogen component with column density $\gtrsim 10^{20} \mathrm{~cm}^{-2}$. Furthermore, in the case of M29 , the He II emission region is hidden from our line of sight, whereas the H I scattering site is seen. 

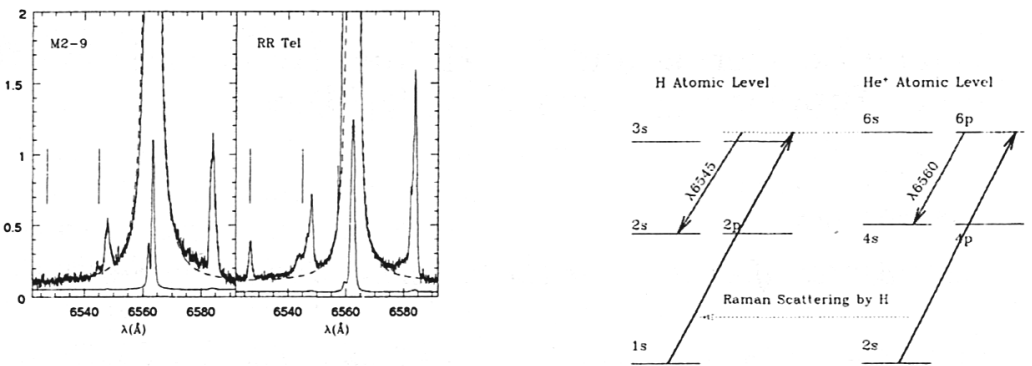

Figure 1. CTIO spectra of M2-9 and RR Tel and the Raman scattering process of He II 1025 by atomic hydrogen. The bars mark the He emission lines at $6527 \AA$ and the Raman scattered line at $6545 \AA$.

\section{Photoionization Calculation and Discussion}

We perform photoionization computations using the code 'Cloudy 94' in order to obtain the size of the He II emission region (Ferland 2001). We assume that the electron density $n_{e}=10^{4} \mathrm{~cm}^{-3}$ and the cosmic abundance. By varying the temperature $T_{*}$ and luminosity $L_{*}$ of the central star we typically obtain the size of the He II emission region $\lesssim 10^{16} \mathrm{~cm}$ when $T_{*} \gtrsim 10^{5} \mathrm{~K}$ and $L_{*} \gtrsim 10^{3} L_{\odot}$. With these parameters, the fluxes of He II 1025 and [N II] 6548 are comparable, which is consistent with the observation. This implies that M2-9 harbors a very hot star obscured by the thick circumstellar region.

The planetary nebula M2-9 should not be classified as a low excitation nebula on the basis of the absence of He emission lines, because they are simply hidden from our line of sight. In the presence of the binary companion, the binary interaction may lead to a hot star responsible for ionization of helium. The Raman scattering nature of the $6545 \AA$ feature is consistent with the Raman scattering origin of the broad $\mathrm{H} \alpha$ wings in M2-9 and in other young planetary nebulae. We may expect that the Raman scattering process can be confirmed with spectropolarimetric observations using large telescopes.

\section{References}

Balick, B. 1989, AJ, 97, 476

Doyle, S., Balick, B. Corradi, H. E., 1995, å, 293, 872

Ferland, G., 2001, Hazy, a brief introduction to Cloudy 94.00

Lee, H. -W., 2000, ApJ, 541, L25

Lee, H. -W., Kang, Y. W. \& Byun, Y. I., 2001, ApJ, 551, L121

Schmid, H. M., 1989, å, 211, L31 